Opt i mizi ng si gnal i nt ensi ty cor rect i on during eval uat i on of hepat i c par enchymal enhancement on gadoxet at e di sodi um enhanced MR : compari son of three methods

\begin{tabular}{|c|c|}
\hline 著者 & 小野田 農 \\
\hline 著者別表示 & Onoda Mnori \\
\hline $\begin{array}{l}\text { j our nal or } \\
\text { publ i cat } \mathrm{i} \text { on } \mathrm{titl} \text { e }\end{array}$ & 博士論文本文Ful I \\
\hline 学位授与番号 & 13301甲第4299号 \\
\hline 学位名 & 博士 (保健学) \\
\hline 学位授与年月日 & $2015-09-28$ \\
\hline URL & ht t p: //hdl . handl e. net /2297/43814 \\
\hline
\end{tabular}




\title{
Optimizing signal intensity correction during evaluation of hepatic parenchymal enhancement on gadoxetate disodium-enhanced MRI: Comparison of three methods
}

\author{
Minori Onoda ${ }^{\mathrm{a}, \mathrm{b}, 1}$, Tomoko Hyodo ${ }^{\mathrm{c}, 1}$, Takamichi Murakami ${ }^{\mathrm{c}, *}$, Masahiro Okada ${ }^{\mathrm{c}, 1}$, \\ Tatsuro Uto $^{\mathrm{a}, 1}$, Masatoshi Hori ${ }^{\mathrm{d}, 2}$, Tosiaki Miyati ${ }^{\mathrm{b}, 3}$ \\ a Department of Radiological Technology, Kinki University Hospital, 377-2 Ohno-Higashi, Osaka-Sayama, Osaka 589-8511, Japan \\ b Division of Health Sciences, Graduate School of Medical Science, Kanazawa University, 5-11-80 Kodatsuno, Kanazawa, Ishikawa 920-0942, Japan \\ ${ }^{\mathrm{c}}$ Department of Radiology, Kinki University Faculty of Medicine, 377-2 Ohno-Higashi, Osaka-Sayama, Osaka 589-8511, Japan \\ d Department of Radiology, Osaka University Graduate School of Medicine, 2-2 Yamadaoka, Suita, Osaka 565-0871, Japan
}

\section{A R T I C L E I N F O}

\section{Article history:}

Received 6 May 2014

Received in revised form 8 November 2014

Accepted 18 November 2014

\section{Keywords:}

Magnetic resonance imaging

Gadoxetate disodium

Liver function tests

\begin{abstract}
A B S T R A C T
Objective: To compare signal intensity (SI) correction using scale and rescale slopes with SI correction using SIs of spleen and muscle for quantifying multiphase hepatic contrast enhancement with Gd-EOBDTPA by assessing their correlation with $\mathrm{T}_{1}$ values generated from Look-Locker turbo-field-echo (LL-TFE) sequence data $\left(E R-\mathrm{T}_{1}\right)$.

Materials and methods: Thirty patients underwent Gd-EOB-DTPA-enhanced magnetic resonance imaging (MRI) in this prospective clinical study. For each patient, breath-hold $\mathrm{T}_{1}$-weighted fat-suppressed three-dimensional (3D) gradient echo sequences (e-THRIVE) were acquired before and 2 (first phase), 10 (second phase), and $20 \mathrm{~min}$ (third phase) after intravenous Gd-EOB-DTPA. Look-Locker turbo-fieldecho (LL-TFE) sequences were acquired before and 1.5 (first phase), 8 (second phase), and 18 min (third phase) postcontrast. The liver parenchyma enhancement ratios (ER) of each phase were calculated using the SI from e-THRIVE sequences (ER-SI) and the $\mathrm{T}_{1}$ values generated from LL-TFE sequence data (ER-T $\mathrm{T}_{1}$ ) respectively. ER-SIs were calculated in three ways: (1) comparing with splenic SI (ER-SI-s), (2) comparing with muscle SI (ER-SI-m), (3) using scale and rescale slopes obtained from DICOM headers (ER-SI-c), to eliminate the effects of receiver gain and scaling. For each of the first, second and third phases, correlation and agreement were assessed between each ER-SI and ER-T $\mathrm{T}_{1}$.

Results: In the first phase, all ER-SIs correlated weakly with ER- ${ }_{1}$. In the second and third phases, ER-SI-c showed a stronger linear correlation with ER-T $\mathrm{T}_{1}\left(r^{2}=0.71-0.72, p<0.01\right)$ than did ER-SI-s $\left(r^{2}=0.37-0.39\right.$, $p<0.01)$ or ER-SI-m $\left(r^{2}=0.30-0.41, p<0.01\right)$.

Conclusion: SI correction using scale and rescale slopes from DICOM data is the most acceptable algorithm for evaluating delayed-phase Gd-EOB-DTPA hepatic enhancement.
\end{abstract}

(c) 2014 Elsevier Ireland Ltd. All rights reserved.

\footnotetext{
* Tel.: +81 72366 0221; fax: +8172367 1685 .

E-mail addresses: onoda@radt.med.kindai.ac.jp (M. Onoda), neneth@m.ehime-u.ac.jp (T. Hyodo), murakami@med.kindai.ac.jp (T. Murakami), okada777@med.u-ryukyu.ac.jp (M. Okada), chuho@med.kindai.ac.jp (T. Uto), mhori@radiol.med.osaka-u.ac.jp (M. Hori),ramiyati@mhs.mp.kanazawa-u.ac.jp (T. Miyati).

1 Tel.: +8172366 0221 .

2 Tel.: +81668795111.

3 Tel.: +81762652500.
}

\section{Introduction}

The assessment of liver function is extremely important for predicting prognosis in patients with hepatic disease [1]. There are several methods of staging liver damage. The Child-Pugh clinical classification is used to determine the prognosis of chronic liver disease, mainly cirrhosis [2]. Slow hepatic clearance of indocyanine green (ICG) is associated with increased surgical risk and poor long-term outcomes [3]. ${ }^{99 \mathrm{~m}} \mathrm{Tc}$-galactosyl human serum albumin is a well-established functional imaging technique for visual and quantitative analysis of radiopharmaceutical tracer uptake in the liver [4]. The drawback of the technique is its limited spatial resolution. 
Gadoxetate disodium-gadolinium ethoxybenzyl diethylenetriamine pentaacetic acid (Gd-EOB-DTPA) has recently come into widespread use in clinical practice. It is a magnetic resonance imaging (MRI) contrast agent that has both extracellular and hepatocyte-specific capabilities. The former characteristic enables dynamic imaging, which reveals the vascularity of the hepatic parenchyma and hepatic lesions, although the dynamic early arterial phase may be hampered due to a low volume compared with other gadolinium agents. The latter characteristic offers hepatocyte/hepatobiliary images - generally acquired $20 \mathrm{~min}$ after administration - that can visualize focal hepatic lesions as areas of hypointensity in the surrounding enhancing parenchyma $[5,6]$.

Studies have investigated the relationship between liver parenchymal enhancement during the hepatobiliary phase of GdEOB-DTPA-enhanced MRI and liver function [7-9]. They have found that the signal intensity (SI) of enhanced liver parenchyma is significantly lower in patients with severe liver dysfunction, because of impaired Gd-EOB-DTPA uptake. SI evaluations also have limitations. One is that the SI is defined on an arbitrary scale that can vary considerably according to prescan calibration. An MR prescan process includes setting the radiofrequency (RF) amplifier and fixing the scale of the SI to acquire optimal images for each scan. In Gd-EOB-DTPA-enhanced examinations, because $\mathrm{T}_{2}$-weighted images $\left(\mathrm{T}_{2} \mathrm{WI}\right)$ and diffusion-weighted images (DWI) are usually acquired between the equilibrium and hepatobiliary phases, a second calibration process is required for hepatobiliary phase image acquisition. For that reason, the SIs in the hepatobiliary phase cannot be directly compared with those of the precontrast images. Another limitation of SI evaluations is that SI can be influenced by system performance and imaging parameters (e.g., magnetic strength, B1-field heterogeneity, repetition time, echo time, longitudinal relaxation time $\left[T_{1}\right]$, and transverse relaxation time $\left[T_{2}\right.$ or $\left.T_{2}{ }^{*}\right]$ ) $[10,11]$. In comparison, the $T_{1}$ value is essentially an absolute value at the same static magnetic field strength, and there is a linear relationship between the relaxation rate ( $R 1$, defined as the reciprocal of the $T_{1}$ value) and the concentration of contrast agent $[12,13]$. This applies to Gd-EOB-DTPA. There are a few studies showing that the $\mathrm{T}_{1}$ value can be used with high accuracy to determine the Child-Pugh classification by evaluating enhancement with GdEOB-DTPA [14]. However, it is difficult to measure $\mathrm{T}_{1}$ values in the abdomen directly because of patient motion (e.g., breathing and heartbeat). Therefore, the SI of liver parenchyma is still widely used for assessing hepatic contrast enhancement. In previous studies, the SI was adjusted for pre- and post-contrast images using the SIs of various reference organs (e.g., spleen $[15,16]$, erector spinae muscle $[16,17])$ or a phantom [18] in the same plane. Another method is to use scaling and rescaling factors obtained from the MR console. The SI displayed on the MR console is affected by scaling of the pixel value. The SI calculated using the scale and rescale slopes is regarded as the "real" SI value (i.e., floating point value), as it is no longer affected by receiver gain or scaling of the displayed image pixel values $[19,20]$. The technique is considered relatively reliable, but it has not been applied to Gd-EOB-DTPA-enhanced MRI.

We sought to compare SIs corrected using scale and rescale slopes with SIs adjusted using the reference SIs of muscle and spleen on multiple phases by quantifying their correlation with $\mathrm{T}_{1}$ values.

\section{Materials and methods}

\subsection{Subjects}

This prospective clinical study followed the principles of the Declaration of Helsinki. The ethics committee of our institution approved the study, and informed consent was obtained in written form from all patients who participated.
Table 1

Pulse sequence parameters for magnetic resonance imaging.

\begin{tabular}{lll}
\hline Parameter & LL-TFE & e-THRIVE \\
\hline TR $(\mathrm{ms})$ & 12.0 & 3.5 \\
TE $(\mathrm{ms})$ & 1.7 & 1.7 \\
Flip angle $\left(^{\circ}\right)$ & 7 & 10 \\
Acquisition & $2 \mathrm{D}$ & $3 \mathrm{D}$ \\
Slice thickness $(\mathrm{mm})$ & 10.0 & 1.5 \\
Acquisition matrix & $112 / 66$ & $320 / 250$ \\
RFOV $(\%)$ & 68 & 80 \\
SENSE factor (direction) & $2.0(\mathrm{AP})$ & $1.9(\mathrm{AP})$ \\
Fat-saturation & No & Yes \\
Acquisition phases & Unenhanced and 1.5, 8, & Unenhanced and 2, 10, \\
& and 18 min after & and 20 min after \\
& administration & administration \\
Acquisition time $(\mathrm{s})$ & 15.0 & 18.5 \\
\hline
\end{tabular}

LL-TFE, Look-Locker turbo field echo; e-THRIVE, enhanced three-dimensional $\mathrm{T}_{1}$ high-resolution isotropic volume excitation; TR, repetition time; TE, echo time; RFOV, rectangular field of view; SENSE, sensitivity encoding.

LL-TFE uses a non-slice-selective inversion pulse as a pre-pulse.

From January 2011 to April 2011, a total of 157 consecutive patients underwent Gd-EOB-DTPA-enhanced MRI for a known or suspected liver tumour. Three patients with Child-Pugh C status were excluded because of insufficient enhancement on MRI during the Gd-EOB-DTPA hepatobiliary phase. Ninety-two patients were excluded for the following reasons: motion artefact due to inadequate breath holding $(n=29)$, and misregistration of slice location between phases $(n=63)$. The remaining 30 patients were evaluated. This group included 15 patients with normal liver function tests, eight with chronic hepatitis, and seven with liver cirrhosis.

\subsection{Data acquisition}

Gd-EOB-DTPA (EOB-Primovist ${ }^{\circledR}$; Bayer Pharma AG, Berlin, Germany) was used as the hepatocyte-specific contrast agent. All patients received Gd-EOB-DTPA at a dose of $0.025 \mathrm{mmol} / \mathrm{kg}$ body weight. It was administered at $2 \mathrm{~mL} / \mathrm{s}$ through an intravenous line placed in an antecubital or cephalic vein. It was flushed with $35 \mathrm{~mL}$ of $0.9 \%$ saline at the same rate.

MRI was performed using a 32-channel phased-array surface coil on a 3.0 T system (Achieva X-series ${ }^{\circledR}$; Philips Medical Systems, Eindhoven, The Netherlands).

In all patients, both breath-hold $\mathrm{T}_{1}$-weighted fat-suppressed three-dimensional (3D) gradient echo sequences (enhanced 3D $\mathrm{T}_{1}$-high resolution isotropic volume excitation [e-THRIVE]) and breath-hold Look-Locker turbo field echo (LL-TFE) sequences were acquired during a single examination. The e-THRIVE sequences were acquired before and at 2 (first phase), 10 (second phase), and 20 min (third phase) after Gd-EOB-DTPA administration. The LL-TFE sequences were acquired before and at 1.5 (first phase), 8 (second phase), and $18 \mathrm{~min}$ (third phase) after Gd-EOB-DTPA administration. An LL-TFE sequence was acquired as a single axial slice at the level of the porta hepatis. Details of each pulse sequence are in Table 1.

\subsection{Image analysis}

The SI displayed on the MR console is affected by scaling of the pixel value. It is calculated during two scaling processes using the scale and rescale slopes. The first scaling process is performed to store data in a limited number of bits. The second is used to display the stored data properly on the MR console. We decoded the displayed SI by referring to scale and rescale slopes on the digital imaging and communication in medicine (DICOM) headers using the Extended MR WorkSpace 2.6 (Philips Medical Systems, Eindhoven, The Netherlands). The scale and rescale slope values were 
acquired from the DICOM tags $(0028,1053)$ and $(2005,100 \mathrm{E})$. The corrected SI was calculated as follows:

Corrected SI $=\frac{\text { Displayed SI }}{\text { scale slope } * \text { rescale slope }}$

The SIs of the liver parenchyma, erector spinae muscle, and spleen were measured on the e-THRIVE precontrast, first-, second-, and third-phase images. The SI for liver parenchyma was obtained by positioning a circular region of interest (ROI) with an area of 50-60 pixels on e-THRIVE images from four imaging series. Two abdominal radiologists with 7 and 12 years of experience, respectively, placed three ROIs on areas of liver parenchyma free of focal hepatic lesions, major branches of the portal or hepatic vein, or imaging artefacts, making sure to place the ROIs at the same positions on the liver for each patient. On visual assessment, no examination displayed a difference in degree of enhancement between left and right lobes in any phase, including the precontrast phase. The mean of the three ROI measurements was recorded as the SI of the liver in each phase. For each of the 2, 10, and 20 min phases, the enhancement ratio based on the SI (ER-SI) for each patient was calculated by each of the following three algorithms: the SI of the liver parenchyma was adjusted by the SI of the spleen (ER-SI-s), the erector spinae muscle (ER-SI-m), and by the scale and rescale slopes (ER-SI-c) $[21,22]$. The following equations show the calculation methods:

$$
\begin{aligned}
& \text { ER-SI-S }=\frac{\left(\mathrm{SI}_{\text {liver }} / \mathrm{SI}_{\text {spleen }}\right)_{\text {post }}-\left(\mathrm{SI}_{\text {liver }} / \mathrm{SI}_{\text {spleen }}\right)_{\text {pre }}}{\left(\mathrm{SI}_{\text {liver }} / \mathrm{SI}_{\text {spleem }}\right)_{\text {pre }}} \\
& \text { ER-SI-m }=\frac{\left(\mathrm{SI}_{\text {liver }} / \mathrm{SI}_{\text {muscle }}\right)_{\text {post }}-\left(\mathrm{SI}_{\text {liver }} / \mathrm{SI}_{\text {muscle }}\right)_{\text {pre }}}{\left(\mathrm{SI}_{\text {liver }} / \mathrm{SI}_{\text {muscle }}\right)_{\text {pre }}} \\
& \text { ER-SI-c }=\frac{\left(\mathrm{SI}_{\text {liver }} /[\mathrm{SS} \cdot \mathrm{RSS})_{\text {post }}-\left(\mathrm{SI}_{\text {liver }} /[\mathrm{SS} \cdot \mathrm{RSS}]\right)_{\text {pre }}\right.}{\left(\mathrm{SI}_{\text {liver }} /[\mathrm{SS} \cdot \mathrm{RSS}]\right)_{\text {pre }}}
\end{aligned}
$$

where $\mathrm{SI}_{\text {liver }}, \mathrm{SI}_{\text {spleen }}$, and $\mathrm{SI}_{\text {muscle }}$ are the signal intensities of the liver parenchyma, spleen parenchyma, and erector spinae muscle; $\mathrm{SS}$ is the scale slope; and RSS is the rescale slope. In each equation, (SI ratio)pre is the SI ratio before Gd-EOB-DTPA administration, and (SI ratio) post is the ratio after Gd-EOB-DTPA administration. To evaluate interobserver variability, measurements were performed independently twice by different observers. The mean of the values of the two observers was used as the measurement for each area.

The Philips Research Integrated Development Environment (PRIDE) $\mathrm{T}_{1}$ fitting tool written in Interactive Data Language 6.3 (IDL, Visual Information Solutions, Boulder CO, USA) was employed to measure the $T_{1}$ values using data from the LL-TFE sequence. Multiple images acquired during signal recovery after a preparation pulse were regarded as parametric images and the $T_{1}$ relaxation time was calculated from every pixel of these images. PRIDE depicts the $\mathrm{T}_{1}$ value on a pixel-by-pixel basis on a colour distribution map. The same ROI method was applied to the $T_{1}$ map of the liver parenchyma on the same slice that was used for the e-THRIVE image. The enhancement ratio based on the $T_{1}$ value $\left(E R-T_{1}\right)$ for each of the $1.5,8$, and 18 min phases was calculated using the following equation:

ER- $T_{1}=\frac{\left(1 / T_{1_{\text {post }}}\right)-\left(1 / T_{1_{\text {pre }}}\right)}{1 / T_{1_{\text {pre }}}}$

where $T_{1 \text { pre }}$ is the $T_{1}$ precontrast value, and $T_{1_{\text {post }}}$ is the $T_{1}$ postcontrast value

\subsection{Statistical analysis}

Interobserver agreement was assessed using Cohen $\kappa$ coefficients for binary measures and the intraclass correlation coefficient (ICC) for continuous measures. Continuous variables are reported as means with standard deviations (SD) and range. The single regression analyses and the Bland-Altman technique were used to evaluate for correlation between ER-T $\mathrm{T}_{1}$ and ER-SI-m, ER-SI-s, or ER-SI-c for the first, second, and third phases. A $p$ value of $<0.01$ was considered statistically significant. The upper and lower limits of agreement were defined as the mean difference \pm 1.96 (SD). In the single regression analyses, the coefficient of determination $\left(r^{2}\right)$ was used to examine the strength of the association between ER-T 1 and ER-SI-s, ER-SI-m, and ER-SI-c. Statistical analyses were performed with a commercial software package (SPSS version 11.0; SPSS, Chicago IL, USA).

\section{Results}

The $T_{1}$ values (mean $\pm S D$ ) for liver parenchyma on the precontrast and at $1.5,8$, and $18 \mathrm{~min}$ after administration (first, second, and third phases, respectively) in the 30 patients were $851 \pm 77 \mathrm{~ms} \quad(702-1058 \mathrm{~ms}), 484 \pm 76 \mathrm{~ms} \quad(357-640 \mathrm{~ms})$, $427 \pm 105 \mathrm{~ms}$ (270-769 ms), and $385 \pm 117 \mathrm{~ms}$ (229-807 ms), respectively (Table 2 ). The $\mathrm{T}_{1}$ value for liver parenchyma decreased with increasing study phase in 29 of the 30 patients. $T_{1}$ maps made it possible to show the differences between precontrast and postcontrast $T_{1}$ values as differences in colour (Fig. 1a). Thus, in 29 patients, liver parenchyma on e-THRIVE was visually the brightest at $20 \mathrm{~min}$ after administration (Fig. 1b). The SI of liver parenchyma was the highest during the second phase. The SIs showed higher SD than $\mathrm{T}_{1}$ values for liver parenchyma in all phases (Table 2). ER$\mathrm{T}_{1}$ and ER-SI-c increased steeply between contrast injection and the acquisition of Phase 1, followed by gradual increases. ER-SI-m increased continuously at the same rate until the end of the examination period. ER-SI-s increased very little during the first phase and then gradually increased. Fig. 2 illustrates a plot of one patient. On imaging analysis, the interobserver variability measure showed good agreement, with ICCs of 0.77-0.99 for continuous measures (Table 3).

Fig. 3 and Table 4 display the relation and agreement between ER-T $_{1}$ and ER-SI-m, ER-SI-s, and ER-SI-c by phase. In the first phase, each of these parameters had a weak (ER-SI-c, $r^{2}=0.15, p<0.05$ ) or a nonsignificant (ER-SI-s, $r^{2}=0.04, p=0.13$; ER-SI-m, $r^{2}=0.04$,

\begin{tabular}{|c|c|c|c|c|}
\hline Parameter & Precontrast & First phase & Second phase & Third phase \\
\hline $\mathrm{T}_{1}$ value of liver $(\mathrm{ms})^{\mathrm{a}}$ & $851 \pm 77(702-1058)$ & $484 \pm 76(357-640)$ & $427 \pm 105(270-769)$ & $385 \pm 117(229-807)$ \\
\hline SI of liver ${ }^{\mathrm{a}}$ & $723 \pm 206(464-1167)$ & $856 \pm 169(516-1133)$ & $951 \pm 201(529-1275)$ & $726 \pm 157(443-1054)$ \\
\hline SI of spleen ${ }^{\mathrm{b}}$ & $715 \pm 159(376-1045)$ & $918 \pm 205(472-1384)$ & $680 \pm 210(216-1092)$ & $452 \pm 180(146-946)$ \\
\hline SI of muscle ${ }^{b}$ & $621 \pm 211(297-1273)$ & $721 \pm 177(291-1005)$ & $502 \pm 193(188-977)$ & $300 \pm 132(134-780)$ \\
\hline
\end{tabular}

Table 2

Summary of the $T_{1}$ value of liver and signal intensity of liver, spleen, and muscle acquired at each time point.

Data are means \pm standard deviations. Numbers in parentheses are ranges.

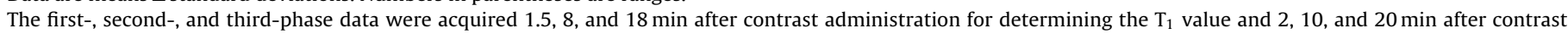
administration for determining signal intensity.

a Two observers individually measured three ROIs.

b Two observers individually measured an ROI. 
(a)
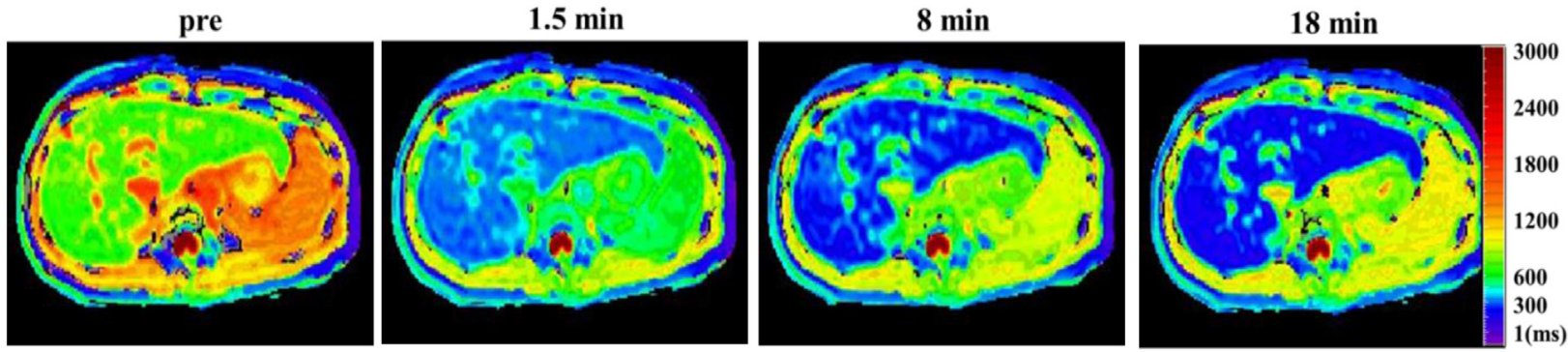

(b)
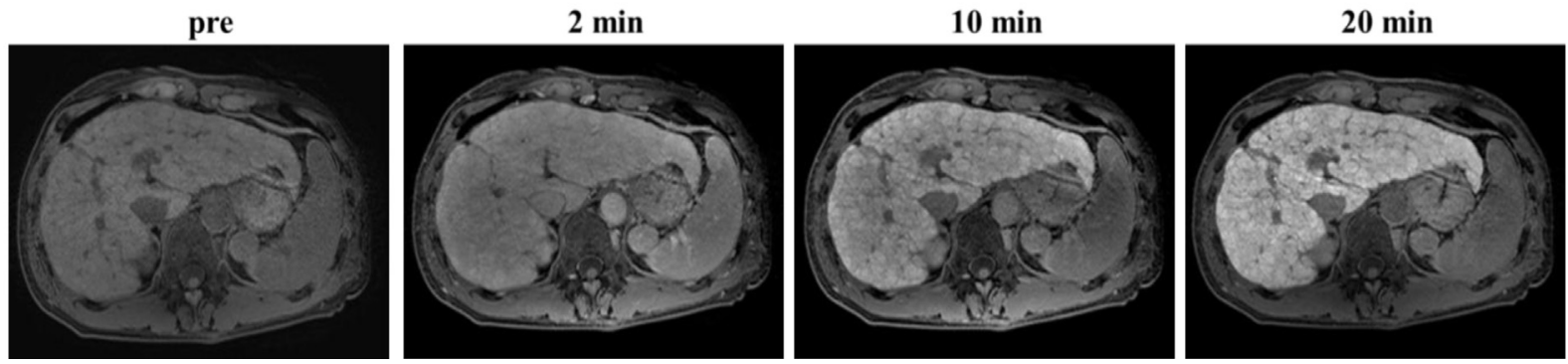

Fig. 1. Sixty-seven-year-old man with Child-Pugh A cirrhosis suspected of having hepatocellular carcinoma. (a) $\mathrm{T}_{1}$ map images generated from Look-Locker turbo field echo (LL-TFE) sequences acquired before and 1.5, 8 , and $18 \mathrm{~min}$ after administration of contrast. The $\mathrm{T}_{1}$ map image shows the differences in $\mathrm{T}_{1}$ values as colour distributions. (b) $\mathrm{T}_{1}$-weighted images using enhanced three-dimensional $\mathrm{T}_{1}$ high-resolution isotropic volume excitation (e-THRIVE) sequences before and 2,10 , and 20 min after contrast administration.

Table 3

Interobserver agreement for the $\mathrm{T}_{1}$ value of liver and signal intensity of liver, spleen, and muscle.

\begin{tabular}{lllll}
\hline Parameter & Precontrast & First phase & Second phase & Third phase \\
\hline $\mathrm{T}_{1}$ value of liver & $0.98(0.96,0.99)$ & $0.99(0.98,1.00)$ & $0.99(0.98,1.00)$ & $0.99(0.98,0.99)$ \\
SI of liver & $0.94(0.88,0.97)$ & $0.85(0.70,0.93)$ & $0.92(0.84,0.96)$ & $0.95(0.68,0.98)$ \\
SI of spleen & $0.83(0.67,0.91)$ & $0.84(0.70,0.92)$ & $0.92(0.83,0.96)$ & $0.94(0.87,0.97)$ \\
SI of muscle & $0.98(0.95,0.99)$ & $0.85(0.70,0.92)$ & $0.84(0.70,0.92)$ & $0.77(0.55,0.89)$ \\
\hline
\end{tabular}

SI, signal intensity.

Data in parentheses are $95 \%$ confidence intervals (CIs).

The first-, second-, and third-phase data were acquired $1.5,8$, and 18 min after contrast administration for determining the $\mathrm{T}_{1}$ value and 2,10 , and 20 min after administration for determining signal intensity.

$p=0.13$ ) correlation with ER- $\mathrm{T}_{1}$. During the second and third phases ER-SI-c showed a stronger linear relationship with ER-T 1 $\left(r^{2}=0.71-0.72, p<0.01\right)$ than did ER-SI-s $\left(r^{2}=0.37-0.39, p<0.01\right)$ or ER-SI-m $\left(r^{2}=0.30-0.41, p<0.01\right)$. Although both ER-T $\mathrm{T}_{1}$ and ER-SI-C

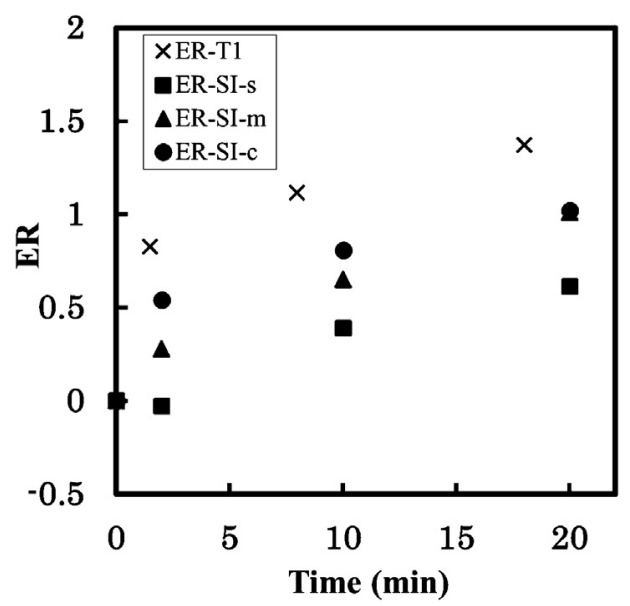

Fig. 2. Sixty-seven-year-old man with Child-Pugh A cirrhosis suspected of having hepatocellular carcinoma. Time-intensity curves for the enhancement ratio (ER) based on the $\mathrm{T}_{1}$ value (ER-T $\mathrm{T}_{1}$ ) and signal intensity. The latter is adjusted with spleen (ER-SI-s) or muscle (ER-SI-m) intensity or the scaling and rescaling factor (ER-SI-c). during the second and third phases showed similar trends in measurements (Table 4), there was a bias between ER-T 1 and ER-SI-c; the variability in disagreement increased as the average increases with fun shape on the Bland-Altman plots (not shown). During the third phase, ER-SI-s, ER-SI-m, and ER-SI-c showed stronger positive linear correlations with ER- $\mathrm{T}_{1}$ than during any other phase.

Table 4

Bland-Altman analysis comparing ER-T ${ }_{1}$ and either ER-SI-m, ER-SI-s, or ER-SI-c at each phase.

\begin{tabular}{llllr}
\hline Parameter & Mean difference & SD & \multicolumn{2}{c}{ Limit of agreement } \\
\cline { 4 - 5 } & & & Lower & Upper \\
\hline ER-T 1 vs. ER-SI-s & & & & \\
1st phase & -0.83 & 0.15 & -1.14 & -0.52 \\
2nd phase & -0.59 & 0.22 & -1.03 & -0.16 \\
3rd phase & -0.59 & 0.29 & -1.17 & -0.01 \\
ER-T 1 vs. ER-SI-m & & & & \\
1st phase & -0.72 & 0.23 & -1.18 & -0.26 \\
2nd phase & -0.35 & 0.22 & -0.79 & 0.09 \\
3rd phase & -0.14 & 0.32 & -0.79 & 0.50 \\
ER-T 1 vs. ER-SI-c & & & & \\
1st phase & -0.34 & 0.16 & -0.66 & -0.01 \\
2nd phase & -0.19 & 0.13 & -0.45 & 0.08 \\
3rd phase & -0.26 & 0.17 & -0.61 & 0.09 \\
\hline
\end{tabular}




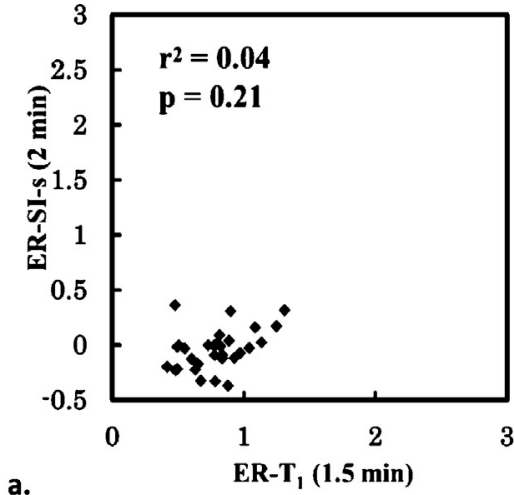

a.
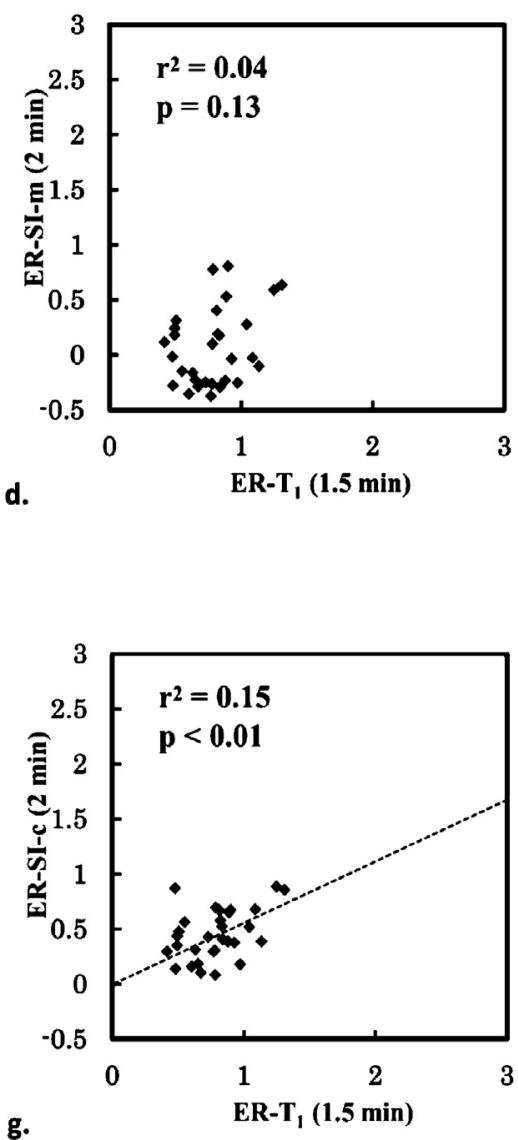
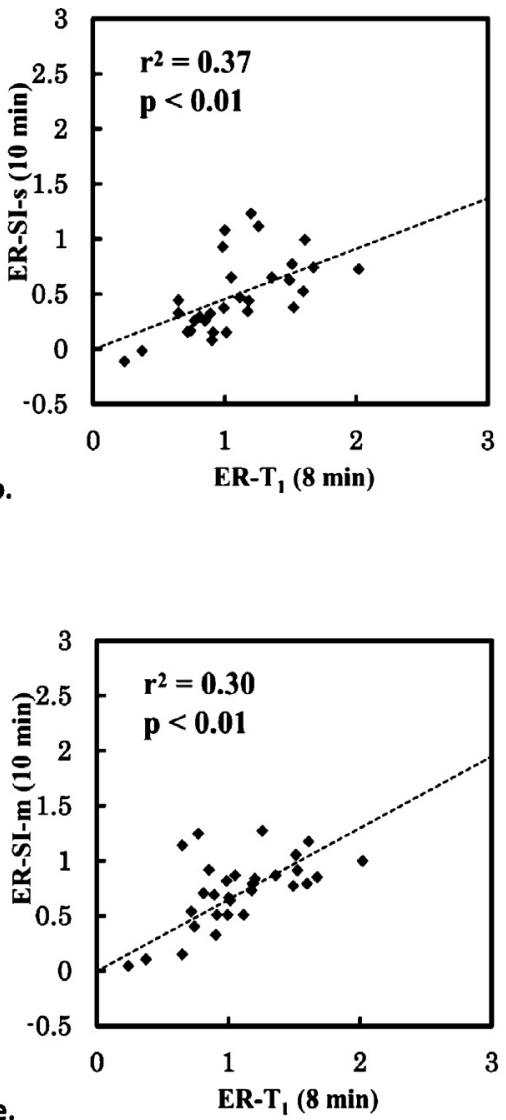

e.

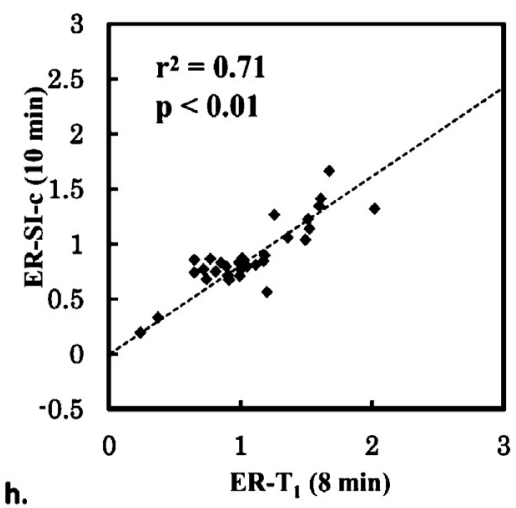

h.

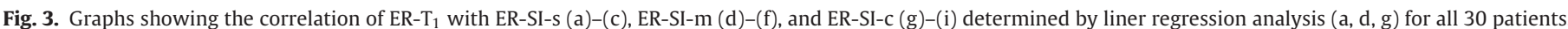

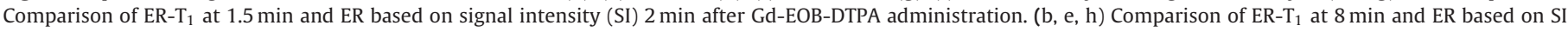
10 min after administration. (c, f, i) Comparison of ER- $\mathrm{T}_{1}$ at $18 \mathrm{~min}$ and ER based on signal intensity 20 min after administration. Dotted lines are the regression lines.

\section{Discussion}

Of the three methods for correcting SI, ER-SI-c showed the strongest positive correlation with ER- $\mathrm{T}_{1}$. The unique features of our study were that the hepatic enhancement SI values were compared with those of the $\mathrm{T}_{1}$ value at three time points after GdEOB-DTPA administration, and SIs were corrected using scale and rescale slopes from the DICOM data. Several studies have shown the relationship between liver function and $T_{1}$ relaxation time. Kamimura et al. showed that the reduction rate of the $\mathrm{T}_{1}$ relaxation time of liver parenchyma could distinguish Child-Pugh A and $B$ from normal liver better than the relative enhancement of the
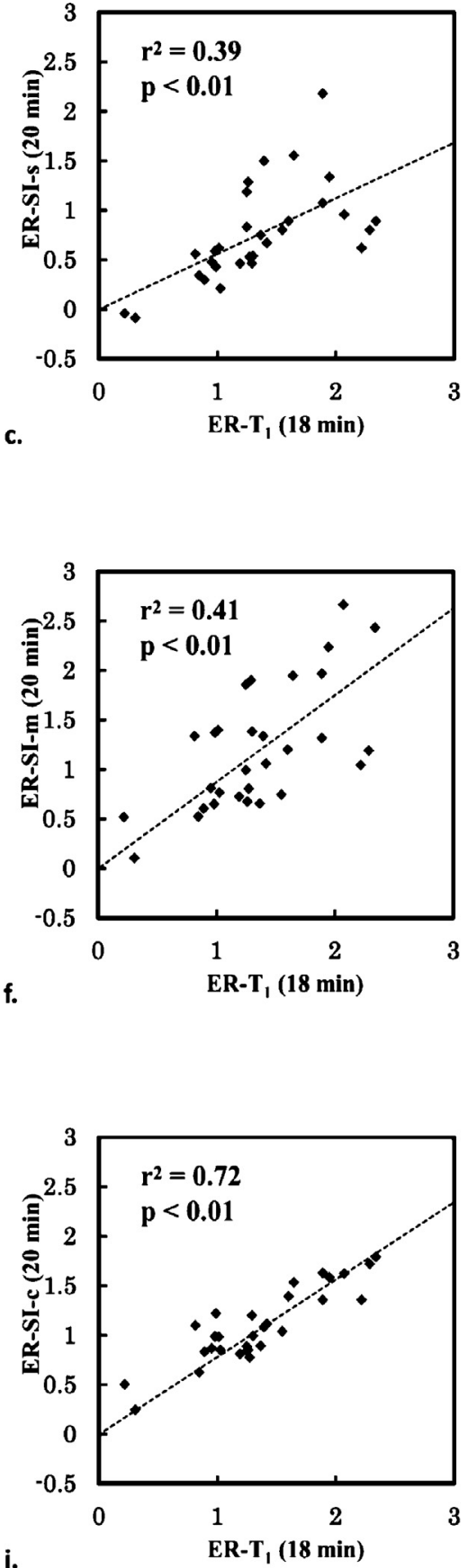

by liner regression analysis (a, d, g) for all 30 patie
ministration. Dotted lines are the regression lines.

liver using SI [23]. In their study, the SI of paravertebral muscle and the spleen were used as the SI reference for liver parenchyma, but the scale and rescale slopes were not used. Also, they calculated the reduction rate of the $\mathrm{T}_{1}$ relaxation time at only one time point (20 min after Gd-EOB-DTPA administration). Because liver enhancement with this method depends on the time needed for uptake and excretion of Gd-EOB-DTPA, we compared the relationship between the SI and $T_{1}$ value indices for hepatic enhancement at three time points after Gd-EOB-DTPA administration.

Whereas almost all of the liver parenchymal enhancement on 20 min e-THRIVE images was the brightest of all phases, the SIs of liver parenchyma at $20 \mathrm{~min}$ were lower than those at $10 \mathrm{~min}$. 
The reason appears to be that the biliary duct shows very high SI on e-THRIVE images at 20 min due to excretion of Gd-EOB-DTPA, so the liver parenchyma SI is adjusted lower. The adjustment of SI is mainly attributed to the scale and rescale slopes. The same explanation applies to the finding that the SIs of spleen and muscle at 20 min were low, thus verifying the significance of SI adjustment for evaluating hepatic enhancement with Gd-EOB-DTPA.

ER-SI-c was significantly correlated with ER- $\mathrm{T}_{1}$ during the hepatobiliary phases (10 and $20 \mathrm{~min}$ postcontrast), suggesting that SI can be used to assess hepatic enhancement with Gd-EOBDTPA in the hepatobiliary phase by making adjustments using the scale and rescale slopes to reduce the inherent problem of SIs varying considerably because of the prescan. Generally, SI of liver on $\mathrm{T}_{1} \mathrm{WI}$ increases when Gd-EOB-DTPA is taken up by liver parenchyma [14]. The linear correlation is most valid for SI with a low contrast agent concentration because $T_{1}$ effects, and not $\mathrm{T}_{2}{ }^{*}$ effects, predominantly affect SI [24]. During the hepatobiliary phase, the relationship between SI and the Gd-EOB-DTPA concentration in liver parenchyma may be linear. However, the Bland-Altman plots indicated a bias between ER-SI-c and ER-T during the hepatobiliary phase. ER-SI-c tended to be lower than ER$\mathrm{T}_{1}$. In this study, SI reduction was observed in some $\mathrm{T}_{1}$ WIs because of other influences (e.g., B1 field heterogeneity). This finding indicates that the adjustments in this study could not completely eliminate the factors potentially influencing SI [25]. Such small differences can lead to large errors during rigorous assessment of the time course of hepatic enhancement (e.g., pharmacokinetic analysis).

Neither ER-SI-s nor ER-SI-m showed a good linear correlation with ER- $\mathrm{T}_{1}$ at any of the time points. It was because, aside from the disadvantage of using SI (i.e., an arbitrary scale), the SIs of spleen and muscle are themselves variable before and after enhancement. Fatty infiltration or mild enhancement of the erector spinae muscle may cause measurement errors [18]. Portal venous pressure, especially in patients with chronic liver disease, can influence splenic arterial blood flow [26]. The fluctuating SI of spleen or muscle might directly influence the ER of liver parenchyma.

At 2 min after administration, each ER based on SI (ER-SI-s, ERSI-m, ER-SI-c) showed a weak or nonsignificant correlation with $E R-T_{1}$. A possible reason for this is the difference in the sequences between LL-TFE and e-THRIVE: e-THRIVE is a three-dimensional $\mathrm{T}_{1}$-weighted gradient echo technique that requires a longer time for data acquisition than LL-TFE. As a result, arterial enhancement curves and peak values differ on a local level, as contrast agent (excited blood protons) may flow off the tissue during acquisition of 3D data in the early phase. Thus, the e-THRIVE sequence may not be applicable to pharmacokinetic analysis, which requires accurate quantitative data concerning hepatic enhancement throughout the examination.

Our study has some limitations. First, correlation between ER$\mathrm{T}_{1}$ and ER-SI might have been hampered because the LL-TFE and e-THRIVE sequences were performed at time points differing by 30-120 s. During this time, Gd-EOB-DTPA uptake by hepatocytes would increase to some degree. We believe, however, that the changes in the enhancement pattern can be evaluated and factored in. Second, we attempted to eliminate any influence of calibration by using SI ratios between liver and spleen or muscle or with scale and rescale slopes. However, we used single-source parallel RF transmission technology, which is split between two ports that are located $90^{\circ}$ apart with horizontal symmetry. The technology tends to cause B1 inhomogeneity in the abdominal region at high magnetic field strength [27]. The consistency of the SI may be improved even more by correcting other factors, including B1-field heterogeneity and fat suppression. Further study is needed in these areas.

\section{Conclusions}

SI correction using scale and rescale slopes from DICOM data is an acceptable algorithm for assessing hepatic enhancement with Gd-EOB-DTPA during the hepatobiliary phase.

\section{Conflict of interest}

The authors declare that they do not have any affiliation with, or financial relationship/interest in, a commercial organization that could pose a conflict of interest.

\section{References}

[1] Botta F, Giannini E, Romagnoli P, Fasoli A, Malfatti F, Chiarbonello B, et al. MELD scoring system is useful for predicting prognosis in patients with liver cirrhosis and is correlated with residual liver function: a European study. Gut 2003;52:134-9

[2] Pugh RN, Murray-Lyon IM, Dawson JL, Pietroni MC, Williams R. Transection of the oesophagus for bleeding oesophageal varices. Br J Surg 1973;60:646-9.

[3] Kaibori M, Matsui Y, Kitade H, Kwon AH, Kamiyama Y. Hepatic resection for hepatocellular carcinoma in severely cirrhotic livers. Hepatogastroenterology 2003;50:491-6.

[4] Kwon AH, Ha-Kawa SK, Uetsuji S, Inoue T, Matsui Y, Kamiyama Y. Preoperative determination of the surgical procedure for hepatectomy using technetium-99m-galactosyl human serum albumin (99mTc-GSA) liver scintigraphy. Hepatology 1997;25:426-9.

[5] Schuhmann-Giampieri G, Schmitt-Willich H, Press WR, Negishi C, Weinmann HJ, Speck U. Preclinical evaluation of Gd-EOB-DTPA as a contrast agent in MR imaging of the hepatobiliary system. Radiology 1992;183:59-64.

[6] Huppertz A, Balzer T, Blakeborough A, Breuer J, A, Giovagnoni G, Heinz-Peer, et al. Improved detection of focal liver lesions at MR imaging: multicenter comparison of gadoxetic acid-enhanced MR images with intraoperative findings. Radiology 2004;230:266-75.

[7] Kim T, Murakami T, Hasuike Y, Gotoh M, Kato N, Takahashi M, et al. Experimental hepatic dysfunction: evaluation by MRI with Gd-EOB-DTPA. J Magn Reson Imaging 1997; 7:683-8.

[8] Shimizu J, Dono K, Gotoh M, Hasuike Y, Kim T, Murakami T, et al. Evaluation of regional liver function by gadolinium-EOB-DTPA-enhanced MR imaging. Dig Dis Sci 1999;44:1330-7.

[9] Tsuda N, Okada M, Murakami T. New proposal for the staging of nonalcoholic steatohepatitis: evaluation of liver fibrosis on Gd-EOB-DTPA-enhanced MRI. Eur J Radiol 2010;73:137-42

[10] Takayama Y, Nishie A, Asayama Y, Ishigami K, Kakihara D, Nakayama T, et al. Image quality of Gd-EOB-DTPA-enhanced magnetic resonance imaging of the liver using dual-source parallel radiofrequency transmission technology: comparison with the post-processing correction method for B1 inhomogeneity-induced signal loss. Eur J Radiol 2012;81:3035-40.

[11] Jackson A. Analysis of dynamic contrast enhanced MRI. Br J Radiol 2004;77(Spec No. 2):S154-66.

[12] Rosen BR, Belliveau JW, Vevea JM, Brady TJ. Perfusion imaging with NMR contrast agents. Magn Reson Med 1990;14:249-65.

[13] Rohrer M, Bauer H, Mintorovitch J, Requardt M, Weinmann HJ. Comparison of magnetic properties of MRI contrast media solutions at different magnetic field strengths. Invest Radiol 2005;40:715-24.

[14] Katsube T, Okada M, Kumano S, Hori M, Imaoka I, Ishii K, et al. Estimation of liver function using $\mathrm{T}_{1}$ mapping on Gd-EOB-DTPA-enhanced magnetic resonance imaging. Invest Radiol 2011;46:277-83.

[15] Motosugi U, Ichikawa T, Tominaga L, Sou H, Sano K, Ichikawa S, et al. Delay before the hepatocyte phase of Gd-EOB-DTPA-enhanced MR imaging: is it possible to shorten the examination time? Eur Radiol 2009;19:2623-9.

[16] Nishie A, Ushijima Y, Tajima T, Asayama Y, Ishigami K, Kakihara D, et al. Quantitative analysis of liver function using superparamagnetic iron oxide- and Gd-EOB-DTPA-enhanced MRI: comparison with Technetium-99m galactosyl serum albumin scintigraphy. Eur J Radiol 2012;81:1100-4.

[17] Katsube T, Okada M, Kumano S, Imaoka I, Kagawa Y, Hori M, et al. Estimation of liver function using $T_{2} *$ mapping on gadolinium ethoxybenzyl diethylenetriamine pentaacetic acid enhanced magnetic resonance imaging. Eur J Radiol 2012;81:1460-4.

[18] Nakamura S, Awai K, Utsunomiya D, Namimoto T, Nakaura T, Morita K, et al. Chronological evaluation of liver enhancement in patients with chronic liver disease at Gd-EOB-DTPA-enhanced 3-T MR imaging: does liver function correlate with enhancement? Jpn J Radiol 2012;30:25-33.

[19] Dietrich O, Raya JG, Reiser MF. Magnetic resonance noise measurements and signal-quantization effects at very low noise levels. Magn Reson Med 2008;60:1477-87

[20] Crelier G, Luechinger R, Buehrer M, Froehlich J, Giese D. Gryotools. User Manual Recon Programming Environment for Scientists; November, 2011.

[21] Saito K, Kotake F, Ito N, Ozuki T, Mikami R, Abe K, et al. Gd-EOB-DTPA enhanced MRI for hepatocellular carcinoma: quantitative evaluation of tumor enhancement in hepatobiliary phase. Magn Reson Med Sci 2005;4:1-9. 
[22] Fujita M, Yamamoto R, Takahashi M, Tsuji T, Yamanaka T, Miyazawa T, et al. Paradoxic uptake of Gd-EOB-DTPA by hepatocellular carcinoma in mice: quantitative image analysis. J Magn Reson Imaging 1997;7:768-70.

[23] Kamimura K, Fukukura Y, Yoneyama T, Takumi K, Tateyama A, Umanodan A, et al. Quantitative evaluation of liver function with $\mathrm{T}_{1}$ relaxation time index on Gd-EOB-DTPA-enhanced MRI: comparison with signal intensity-based indices. J Magn Reson Imaging 2013;40:884-9.

[24] Kirsch JE. Basic principles of magnetic resonance contrast agents. Top Magn Reson Imaging 1991;3:1-18.

[25] de Bazelaire C, Rofsky NM, Duhamel G, Zhang J, Michaelson MD, George $D$, et al. Combined $T_{2}^{*}$ and $T_{1}$ measurements for improved perfusion and permeability studies in high field using dynamic contrast enhancement. Eur Radiol 2006;16:2083-91.

[26] Kaufman S, Levasseur J. Effect of portal hypertension on splenic blood flow intrasplenic extravasation and systemic blood pressure. Am J Physiol Regul Integr Comp Physiol 2003;284:R1580-5.

[27] Takayama Y, Nishie A, Asayama Y, Ishigami K, Kakihara D, Nakayama $\mathrm{T}$, et al. Image quality of Gd-EOB-DTPA-enhanced magnetic resonance imaging of the liver using dual-source parallel radiofrequency transmission technology: comparison with the post-processing correction method for B1 inhomogeneity-induced signal loss. Eur J Radiol 2012;81: 3035-40. 